\title{
Some subordination results associated with generalized Srivastava-Attiya operator
}

Adel A Attiya ${ }^{1,2^{*}}$ and Ali H Hakami ${ }^{3,4}$

\section{"Correspondence:}

aattiy@mans.edu.eg

1 Department of Mathematics,

Faculty of Science, University of

Mansoura, Mansoura, 35516, Egypt

${ }^{2}$ Current address: Department of

Mathematics, Faculty of Science,

University of Hail, Hail, Saudi Arabia

Full list of author information is

available at the end of the article

\begin{abstract}
The operator $\int_{s, b}(f)$ was introduced in (Srivastava and Attiya in Integral Transforms Spec. Funct. 18(3-4): 207-216, 2007), which makes a connection between Geometric Function Theory and Analytic Number Theory. In this paper, we use the techniques of differential subordination to investigate some classes of admissible functions associated with the generalized Srivastava-Attiya operator in the open unit disc $\mathbb{U}=\{z \in \mathbb{C}:|z|<1\}$.
\end{abstract}

MSC: $30 \mathrm{C} 80 ; 30 \mathrm{C} 10 ; 11 \mathrm{M} 35$

Keywords: analytic functions; differential subordination; Hurwitz-Lerch zeta function; Hadamard product; Srivastava-Attiya operator

\section{Introduction}

Let $A(p)$ denote the class of functions $f(z)$ of the form

$$
f(z)=z^{p}+\sum_{k=1}^{\infty} a_{k+p} z^{k+p}
$$

which are analytic in the open unit disc $\mathbb{U}=\{z \in \mathbb{C}:|z|<1\}$. Also, let $A=A(1)$.

We begin by recalling that a general Hurwitz-Lerch Zeta function $\Phi(z, s, b)$ defined by (cf., e.g., [1, p.121 et seq.])

$$
\Phi(z, s, b)=\sum_{k=0}^{\infty} \frac{z^{k}}{(k+b)^{s}}
$$

$\left(b \in \mathbb{C} \backslash \mathbb{Z}_{0}^{-}, \mathbb{Z}_{0}^{-}=\mathbb{Z}^{-} \cup\{0\}=\{0,-1,-2, \ldots\}, s \in \mathbb{C}\right.$ when $z \in \mathbb{U}, \operatorname{Re}(s)>1$ when $\left.|z|=1\right)$, which contains important functions of the Analytic Number Theory.

Several properties of $\Phi(z, s, b)$ can be found in many papers, for example, Choi et al. [2], Ferreira and López [3], Gupta et al. [4] and Luo and Srivastava [5]. See, also Kutbi and Attiya [6, 7], Srivastava and Attiya [8] and Owa and Attiya [9].

Srivastava and Attiya [8] introduced the operator $J_{s, b}(f)(f \in A)$, which makes a connection between Geometric Function Theory and Analytic Number Theory, defined by

$$
\begin{aligned}
& J_{s, b}(f)(z)=G_{s, b}(z) * f(z) \\
& \quad\left(z \in \mathbb{U} ; f \in A ; b \in \mathbb{C} \backslash \mathbb{Z}_{0}^{-} ; s \in \mathbb{C}\right),
\end{aligned}
$$

@ 2013 Attiya and Hakami; licensee Springer. This is an Open Access article distributed under the terms of the Creative Commons Attribution License (http://creativecommons.org/licenses/by/2.0), which permits unrestricted use, distribution, and reproduction in any medium, provided the original work is properly cited. 
where

$$
G_{s, b}(z)=(1+b)^{s}\left[\Phi(z, s, b)-b^{-s}\right]
$$

and $*$ denotes the Hadamard product (or convolution).

Furthermore, Srivastava and Attiya [8] showed that

$$
J_{s, b}(f)(z)=z+\sum_{k=2}^{\infty}\left(\frac{1+b}{k+b}\right)^{s} a_{k} z^{k} \quad(f \in A) .
$$

As special cases of $J_{s, b}(f)(f \in A)$, Srivastava and Attiya [8] introduced the following identities:

$$
\begin{aligned}
& J_{0, b}(f)(z)=f(z), \\
& J_{1,0}(f)(z)=A(f)(z), \\
& J_{1,1}(f)(z)=L(f)(z), \\
& J_{1, \gamma}(f)(z)=L_{\gamma}(f)(z) \quad(\gamma \text { real; } \gamma>-1),
\end{aligned}
$$

and

$$
J_{\sigma, 1}(f)(z)=I^{\sigma}(f)(z) \quad(\sigma \text { real; } \sigma>0),
$$

where the operators $A(f)$ and $L(f)$ are the integral operators introduced earlier by Alexander [10] and Libera [11], respectively, $L_{\gamma}(f)$ is the generalized Bernardi operator, $L_{\gamma}(f)$ $(\gamma \in \mathbb{N}=\{1,2, \ldots\})$ introduced by Bernardi [12] and $I^{\sigma}(f)$ is the Jung-Kim-Srivastava integral operator introduced by Jung et al. [13].

Moreover, in [8], Srivastava and Attiya defined the operator $J_{s, b}(f)(f \in A)$ for $b \in \mathbb{C} \backslash \mathbb{Z}^{-}$, by using the following relationship:

$$
J_{s, 0}(f)(z)=\lim _{b \rightarrow 0} J_{s, b}(f)(z)
$$

Some applications of the operator $J_{s, b}(f)$ to certain classes in Geometric Function Theory can be found in [14-16] and [17].

Liu [15] defined the generalized Srivastava-Attiya operator as follows:

$$
\begin{gathered}
J_{s, b}^{p}(f)(z)=z^{p}+\sum_{k=1}^{\infty}\left(\frac{1+b}{k+1+b}\right)^{s} a_{k+p} z^{k+p} \\
\left(z \in \mathbb{U} ; f \in A(p) ; b \in \mathbb{C} \backslash \mathbb{Z}_{0}^{-} ; s \in \mathbb{C}\right) .
\end{gathered}
$$

Now, we define the function $G_{s, b, t}$ by

$$
\begin{gathered}
G_{s, b, t}=1+z(t+b)^{s} \Phi(z, s, 1+t+b) \\
\left(z \in \mathbb{U} ; b \in \mathbb{C} \backslash \mathbb{Z}_{0}^{-} ; s \in \mathbb{C} ; t \in \mathbb{R}\right),
\end{gathered}
$$


we denote by

$$
\mathcal{J}_{s, b}^{t}(f): A(p) \longrightarrow A(p)
$$

the operator defined by

$$
\begin{aligned}
& \mathcal{J}_{s, b}^{t}(f)(z)=z^{p} G_{s, b, t} * f(z) \\
& \quad\left(z \in \mathbb{U} ; f \in A(p) ; b \in \mathbb{C} \backslash \mathbb{Z}_{0}^{-} ; s \in \mathbb{C} ; t \in \mathbb{R}\right),
\end{aligned}
$$

where $*$ denotes the convolution or Hadamard product.

We note that

$$
\mathcal{J}_{s, b}^{t}(f)(z)=z^{p}+\sum_{k=1}^{\infty}\left(\frac{t+b}{k+t+b}\right)^{s} a_{k+p} z^{k+p} \quad(z \in \mathbb{U})
$$

and

$$
\mathcal{J}_{s, b}^{1}(f)=J_{s, b}^{p}(f)
$$

Moreover, let $\mathbb{D}$ be the set of analytic functions $q(z)$ and injective on $\bar{U} \backslash E(q)$, where

$$
E(q)=\left\{\zeta \in \partial \mathbb{U}: \lim _{z \rightarrow \zeta} q(z)=\infty\right\}
$$

and $q^{\prime}(z) \neq 0$ for $\zeta \in \partial \mathbb{U} \backslash E(q)$. Further, let $\mathbb{D}_{a}=\{q(z) \in \mathbb{D}: q(0)=a\}$.

In our investigations, we need the following definitions and theorem.

Definition 1.1 Let $f(z)$ and $F(z)$ be analytic functions. The function $f(z)$ is said to be $s u b$ ordinate to $F(z)$, written $f(z) \prec F(z)$, if there exists a function $w(z)$ analytic in $\mathbb{U}$, with $w(0)=0$ and $|w(z)| \leq 1$, and such that $f(z)=F(w(z))$. If $F(z)$ is univalent, then $f(z) \prec F(z)$ if and only if $f(0)=F(0)$ and $f(\mathbb{U}) \subset F(\mathbb{U})$.

Definition 1.2 Let $\Psi: \mathbb{C}^{2} \times \mathbb{U} \rightarrow \mathbb{C}$ be analytic in domain $\mathbb{D}$, and let $h(z)$ be univalent in $\mathbb{U}$. If $p(z)$ is analytic in $\mathbb{U}$ with $\left(p(z), z p^{\prime}(z)\right) \in \mathbb{D}$ when $z \in \mathbb{U}$, then we say that $p(z)$ satisfies a first-order differential subordination if:

$$
\Psi\left(p(z), z p^{\prime}(z) ; z\right) \prec h(z) \quad(z \in \mathbb{U}) .
$$

The univalent function $q(z)$ is called dominant of the differential subordination (1.13), if $p(z) \prec q(z)$ for all $p(z)$ satisfies (1.13), if $\tilde{q}(z) \prec q(z)$ for all dominant of (1.13), then we say that $\tilde{q}(z)$ is the best dominant of (1.13).

Definition $1.3[18$, p.27] Let $\Omega$ be a set in $\mathbb{C}, q \in \mathbb{D}$ and $n \in \mathbb{N}=\{1,2, \ldots\}$. The class of admissible function $\Psi_{n}[\Omega, q]$ consists of those functions $\psi: \mathbb{C}^{3} \times \mathbb{U} \rightarrow \mathbb{C}$ that satisfy the admissibility condition $\psi(r, s, \tau ; z) \notin \Omega$ whenever $r=q(\zeta), s=k \zeta q^{\prime}(\zeta)$, and

$$
\operatorname{Re}\left(\frac{\tau}{s}+1\right) \geq k \operatorname{Re}\left(\frac{\zeta q^{\prime \prime}(\zeta)}{q^{\prime}(\zeta)}+1\right) \quad(z \in \mathbb{U} ; \zeta \in \overline{\mathbb{U}} \backslash E(q) ; k \geq n)
$$

We write $\Psi_{1}[\Omega, q]$ as $\Psi[\Omega, q]$. 
In particular, when $q(z)=\frac{M(M z+a)}{M+\bar{a} z}$ with $M>0$ and $|a|<M$, then $q(\mathbb{U})=\mathbb{U}_{M}:=\{w:|w|<$ $M\}, q(0)=a, E(q)=0$ and $q \in \mathbb{D}$. In this case, we set $\Psi_{n}[\Omega, M, a]:=\Psi_{n}[\Omega, q]$ and in the special case when the set $\Omega=\mathbb{U}_{M}$, the class is simply denoted by $\Psi_{n}[M, a]$.

Theorem $1.1\left[18\right.$, p.27] Let $\Psi \in \Psi_{n}[\Omega, q]$ with $q(0)=$ a. If the analytic function $p(z)=a+$ $\sum_{k=n}^{\infty} a_{k} z^{k}$ satisfies

$$
\Psi\left(p(z), z p^{\prime}(z), z^{2} p^{\prime \prime}(z) ; z\right) \in \Omega \quad(z \in \mathbb{U})
$$

then $p(z) \prec q(z)$.

\section{Some subordination results with $\mathcal{J}_{s, b}^{t}$}

Definition 2.1 Let $\Omega$ be a set in $\mathbb{C}$ and $q(z) \in \mathbb{D} \cap A_{p}$. The class of admissible functions $\Phi[\Omega, q]$ consists of those functions $\phi: \mathbb{C}^{3} \times \mathbb{U} \rightarrow \mathbb{C}$ that satisfy the admissibility condition:

$$
\phi(u, v, w ; z) \notin \Omega
$$

whenever

$$
\begin{aligned}
& u=q(\varsigma), \quad v=\frac{k \varsigma q^{\prime}(\varsigma)+(t+b-p) q(\varsigma)}{t+b} \\
& \operatorname{Re}\left(\frac{(t+b)^{2} w-(t+b-p)^{2} u}{(t+b) v-(t+b-p) u}-2(t+b-p)\right) \geq k \operatorname{Re}\left(\frac{\varsigma q^{\prime \prime}(\varsigma)}{q^{\prime}(\varsigma)}+1\right)
\end{aligned}
$$

where $z \in \mathbb{U}, \zeta \in \partial \mathbb{U} \backslash E(q)$ and $k \geq p$.

Theorem 2.1 Let $\phi \in \Phi[\Omega, q]$. If $f(z) \in A_{p}$ satisfies

$$
\left\{\phi\left(\mathcal{J}_{s+1, b}^{t}(z), \mathcal{J}_{s, b}^{t}(z), \mathcal{J}_{s-1, b}^{t}(z) ; z\right)\right\} \subset \Omega \quad(z \in \mathbb{U}),
$$

then

$$
\mathcal{J}_{s+1, b}^{t} \prec q(z)
$$

Proof Let us define the analytic function $p(z)$ as

$$
p(z)=\mathcal{J}_{s+1, b}^{t} f(z) \quad(z \in \mathbb{U}) .
$$

Using the definition of $\mathcal{J}_{s, b}^{t}$, we can prove that

$$
z\left(\mathcal{J}_{s+1, b}^{t} f(z)\right)^{\prime}=(t+b) \mathcal{J}_{s, b}^{t} f(z)-(t+b-p) \mathcal{J}_{s+1, b}^{t} f(z)
$$

then we get

$$
\mathcal{J}_{s, b}^{t} f(z)=\frac{z p^{\prime}(z)+(t+b-p) p(z)}{(t+b)}
$$


which implies

$$
\mathcal{J}_{s-1, b}^{t} f(z)=\frac{z^{2} p^{\prime \prime}(z)+(2(t+b-p)+1) z p^{\prime}(z)+(t+b-p)^{2} p(z)}{(t+b)^{2}} .
$$

Let us define the parameters $u, v$ and $w$ as

$$
u=r, \quad v=\frac{s+(t+b-p) r}{(t+b)} \quad \text { and } \quad w=\frac{\tau+(2(t+b-p)+1) s+(t+b-p)^{2} r}{(t+b)^{2}}
$$

Now, we define the transformation

$$
\begin{aligned}
& \psi: \mathbb{C}^{2} \times \mathbb{U} \rightarrow \mathbb{C}, \\
& \psi(r, s, \tau, z)=\phi(u, v, w ; z),
\end{aligned}
$$

by using the relations (2.3), (2.5), (2.6) and (2.8), we have

$$
\psi\left(p(z), z p^{\prime}(z), z^{2} p^{\prime \prime}(z) ; z\right)=\phi\left(\mathcal{J}_{s+1, b}^{t} f(z), \mathcal{J}_{s, b}^{t}(z), \mathcal{J}_{s-1, b}^{t}(z) ; z\right)
$$

Therefore, we can rewrite (2.1) as

$$
\psi\left(p(z), z p^{\prime}(z), z^{2} p^{\prime \prime}(z) ; z\right) \in \Omega
$$

Then the proof is completed by showing that the admissibility condition for $\phi \in \Phi[\Omega, q]$ is equivalent to the admissibility condition for $\Psi$ as given in Definition 1.3.

Since

$$
\frac{\tau}{s}+1=\frac{(t+b)^{2} w-(t+b-p)^{2} u}{(t+b) v-(t+b-p) u}-2(t+b-p) .
$$

Therefore, $\psi \in \Psi[\Omega, q]$. Also, by Theorem 1.1, $p(z) \prec q(z)$.

If $\Omega \neq \mathbb{C}$ is a simply connected domain, then $\Omega=h(\mathbb{U})$ for some conformal mapping $h(z)$ of $\mathbb{U}$ onto $\Omega$. In this case the class $\Phi[h(\mathbb{U}), q]$ is written as $\Phi[h, q]$.

The following theorem is a direct consequence of Theorem 2.1 .

Theorem 2.2 Let $\phi \in \Phi[h, q]$. If $f(z) \in A(p)$ satisfies the following subordination relation:

$$
\phi\left(\mathcal{J}_{s+1, b}^{t}(z), \mathcal{J}_{s, b}^{t}(z), \mathcal{J}_{s-1, b}^{t}(z) ; z\right) \prec h(z) \quad(z \in \mathbb{U}),
$$

then

$$
\mathcal{J}_{s+1, b}^{t} \prec q(z)
$$

The next corollary is an extension of Theorem 2.2 to the case where the behavior of $q(z)$ on $\partial \mathbb{U}$ is not known. 
Corollary 2.1 Let $\Omega \subset \mathbb{C}$ and let $q(z)$ be univalent in $\mathbb{U}, q(0)=0$. Let $\phi \in \Phi\left[\Omega, q_{\rho}\right]$ for some $\rho \in(0,1)$ where $q_{\rho}(z)=q(\rho z)$. If $f(z) \in A(p)$ satisfies

$$
\phi\left(\mathcal{J}_{s+1, b}^{t}(z), \mathcal{J}_{s, b}^{t}(z), \mathcal{J}_{s-1, b}^{t}(z) ; z\right) \in \Omega \quad(z \in \mathbb{U})
$$

then

$$
\mathcal{J}_{s+1, b}^{t} \prec q(z)
$$

Proof By using Theorem 2.1, we have $\mathcal{J}_{s+1, b}^{t} \prec q_{\rho}(z)$. Then we obtain the result from $q_{\rho}(z) \prec q(z)$.

Theorem 2.3 Let $h(z)$ and $q(z)$ be univalent in $\mathbb{U}$, with $q(0)=0$ and set $q_{\rho}(z)=q(\rho z)$ and $h_{\rho}(z)=h(\rho z)$. Let $\phi: \mathbb{C}^{3} \times \mathbb{U} \rightarrow \mathbb{C}$ satisfy one of the following conditions:

(1) $\phi \in \Phi\left[h, q_{\rho}\right]$ for some $\rho \in(0,1)$, or

(2) there exists $\rho_{0} \in(0,1)$ such that $\phi \in \Phi\left[h_{\rho}, q_{\rho}\right]$ for all $\rho \in\left(\rho_{0}, 1\right)$.

Then

$$
\mathcal{J}_{s+1, b}^{t} f(z) \prec q(z) \quad(f(z) \in A(p)) .
$$

Proof The proof is similar to the proof of [18, Theorem 2.3d, p.30], therefore, we omitted it.

Theorem 2.4 Let $h(z)$ be univalent in $\mathbb{U}$. Let $\phi: \mathbb{C}^{3} \times \mathbb{U} \rightarrow \mathbb{C}$. Suppose that the differential equation

$$
\begin{aligned}
& \phi\left(q(z), \frac{z q^{\prime}(z)+(t+b-p) q(z)}{(t+b)},\right. \\
& \left.\quad \frac{z^{2} q^{\prime \prime}(z)+(2(t+b-p)+1) z q^{\prime}(z)+(t+b-p)^{2} q(z)}{(t+b)^{2}} ; z\right)=h(z),
\end{aligned}
$$

has a solution $q(z)$ with $q(0)=0$ and satisfies one of the following conditions:

(1) $q(z) \in \mathbb{D}_{0}$ and $\phi \in \Phi[h, q]$,

(2) $q(z)$ is univalent in $\mathbb{U}$ and $\phi \in \Phi\left[h, q_{\rho}\right]$ for some $\rho \in(0,1)$, or

(3) $q(z)$ is univalent in $\mathbb{U}$ and there exists $\rho_{0} \in(0,1)$ such that $\phi \in \Phi\left[h_{\rho}, q_{\rho}\right]$ for all $\rho \in\left(\rho_{0}, 1\right)$.

Then

$$
\mathcal{J}_{s+1, b}^{t} f(z) \prec q(z) \quad(f(z) \in A(p)),
$$

and $q(z)$ is the best dominant.

Proof Following the same proof in [18, Theorem 2.3e, p.31], we deduce from Theorems 2.2 and 2.3 that $q(z)$ is a dominant of (2.13). Since $q(z)$ satisfies (2.12), it is also a solution of (2.11) and, therefore, $q(z)$ will be dominated by all dominants. Hence, $q(z)$ is the best dominant. 
In the case $q(z)=M z, M>0$ and in view of the Definition 2.1, the class of admissible functions $\Phi[\Omega, q]$ denoted by $\Phi[\Omega, M]$ is defined below.

Definition 2.2 Let $\Omega$ be a set in $\mathbb{C}$ and $M>0$. The class of admissible functions $\Phi[\Omega, M]$ consists of those functions $\phi: \mathbb{C}^{3} \times \mathbb{U} \rightarrow \mathbb{C}$ that satisfy the admissibility condition

$$
\phi\left(M e^{i \theta}, \frac{k+t+b-p}{t+b} M e^{i \theta}, \frac{L+\left((2 t+2 b-2 p+1) k+(t+b-p)^{2}\right) M e^{i \theta}}{(t+b)^{2}} ; z\right) \notin \Omega,
$$

where $z \in \mathbb{U}$, and $\operatorname{Re}\left(L e^{-i \theta}\right) \geq(k-1) k M$ for all real $\theta$ and $k \geq p$.

Corollary 2.2 Let $\phi \in \Phi[\Omega, M]$. If $f(z) \in A(p)$ satisfies

$$
\phi\left(\mathcal{J}_{s+1, b}^{t}(z), \mathcal{J}_{s, b}^{t}(z), \mathcal{J}_{s-1, b}^{t}(z) ; z\right) \in \Omega \quad(z \in \mathbb{U})
$$

then

$$
\left|\mathcal{J}_{s+1, b}^{t}(z)\right|<M
$$

In the case $\Omega=q(\mathbb{U})=\{\omega:|w|<M\}$, for simplification, we denote by $\Phi[M]$ to the class $\Phi[\Omega, M]$.

Corollary 2.3 Let $\phi \in \Phi[M]$.Iff $(z) \in A(p)$ satisfies

$$
\left|\phi\left(\mathcal{J}_{s+1, b}^{t}(z), \mathcal{J}_{s, b}^{t}(z), \mathcal{J}_{s-1, b}^{t}(z) ; z\right)\right|<M \quad(z \in \mathbb{U}),
$$

then

$$
\left|\mathcal{J}_{s+1, b}^{t}(z)\right|<M \text {. }
$$

Corollary 2.4 Let $M>0$ and $\operatorname{Re}(b)>p-t$. If $f(z) \in A(p)$ satisfies

$$
\begin{aligned}
& \left|(t+b-p)^{2} \mathcal{J}_{s+1, b}^{t}(z)+(t+b) \mathcal{J}_{s, b}^{t}(z)-(t+b)^{2} \mathcal{J}_{s-1, b}^{t}(z)\right| \\
& \quad<[p(p-1)+(2 p-1)(t-p+\operatorname{Re}(b))],
\end{aligned}
$$

then

$$
\left|\mathcal{J}_{s+1, b}^{t}(z)\right|<M
$$

Proof In Corollary 2.2, taking $\phi(u, v, w ; z)=(t+b-p)^{2} u-(t+b) v-(t+b)^{2} w$ and $\Omega=h(\mathbb{U})$ where $h(z)=[p(p-1)+(2 p-1)(t-p+\operatorname{Re}(b))] M z$.

Since

$$
\begin{aligned}
& \left|\phi\left(M e^{i \theta}, \frac{k+t+b-p}{t+b} M e^{i \theta}, \frac{L+\left((2 t+2 b-2 p+1) k+(t+b-p)^{2}\right) M e^{i \theta}}{(t+b)^{2}} ; z\right)\right| \\
& \quad=\mid(t+b-p)^{2} M e^{i \theta}-(k+t+b-p) M e^{i \theta}
\end{aligned}
$$




$$
\begin{aligned}
& -\left[L+\left((2 t+2 b-2 p+1) k+(t+b-p)^{2}\right) M e^{i \theta}\right] \mid \\
= & \left|L+(2 k-1)(t+b-p) M e^{i \theta}\right| \\
\geq & \operatorname{Re}\left(L e^{-i \theta}\right)+(2 k-1) M \operatorname{Re}(t+b-p) \\
\geq & k(k-1) M+(2 k-1) M(t-p+\operatorname{Re}(b)) \\
\geq & {[p(p-1)+(2 p-1)(t-p+\operatorname{Re}(b))] M . }
\end{aligned}
$$

Therefore, $\phi \in \Phi[\Omega, M]$ satisfies the admissible condition (2.14). Then we have the theorem by Corollary 2.2.

Definition 2.3 Let $\Omega$ be a set in $\mathbb{C}$ and $q(z) \in \mathbb{D}_{0} \cap A$. The class of admissible functions $\Phi_{1}[\Omega, M]$ consists of those functions: $\mathbb{C}^{3} \times \mathbb{U} \rightarrow \mathbb{C}$ that satisfy the admissibility condition

$$
\phi(u, v, w ; z) \notin \Omega,
$$

whenever

$$
\begin{aligned}
& u=q(\varsigma), \quad v=\frac{k \varsigma q^{\prime}(\varsigma)+(t+b-1) q(\varsigma)}{t+b}, \\
& \operatorname{Re}\left(\frac{(t+b)^{2} w-(t+b-1)^{2} u}{(t+b) v-(t+b-1) u}-2(t+b-1)\right) \geq k \operatorname{Re}\left(\frac{\varsigma q^{\prime \prime}(\varsigma)}{q^{\prime}(\varsigma)}+1\right)
\end{aligned}
$$

where $z \in \mathbb{U}, \zeta \in \partial \mathbb{U} \backslash E(q)$ and $k \geq 1$.

Theorem 2.5 Let $\phi \in \Phi_{1}[\Omega, q]$. If $f(z) \in A_{p}$ satisfies

$$
\left\{\phi\left(\frac{\mathcal{J}_{s+1, b}^{t}(z)}{z^{p-1}}, \frac{\mathcal{J}_{s, b}^{t}(z)}{z^{p-1}}, \frac{\mathcal{J}_{s-1, b}^{t}(z)}{z^{p-1}} ; z\right)\right\} \subset \Omega \quad(z \in \mathbb{U})
$$

then

$$
\frac{\mathcal{J}_{s+1, b}^{t}}{z^{p-1}} \prec q(z)
$$

Proof Let us define the analytic function $p(z)$ as

$$
p(z)=\frac{\mathcal{J}_{s+1, b}^{t} f(z)}{z^{p-1}} \quad(z \in \mathbb{U}) .
$$

By using (2.4), we have

$$
\frac{\mathcal{J}_{s, b}^{t} f(z)}{z^{p-1}}=\frac{z p^{\prime}(z)+(t+b-1) p(z)}{(t+b)}
$$

which implies

$$
\frac{\mathcal{J}_{s-1, b}^{t} f(z)}{z^{p-1}}=\frac{z^{2} p^{\prime \prime}(z)+(2(t+b)-1) z p^{\prime}(z)+(t+b-1)^{2} p(z)}{(t+b)^{2}} .
$$


Define the parameters $u, v$ and $w$ as

$$
u=r, \quad v=\frac{s+(t+b-1) r}{(t+b)} \quad \text { and } \quad w=\frac{\tau+(2(t+b)-1) s+(t+b-1)^{2} r}{(t+b)^{2}}
$$

now, we define the transformation

$$
\begin{aligned}
\psi: \mathbb{C}^{2} \times \mathbb{U} \rightarrow \mathbb{C} \\
\psi(r, s, \tau ; z)=\phi(u, v, w ; z),
\end{aligned}
$$

by using the relations (2.3), (2.5), (2.6) and (2.8), we have

$$
\psi\left(p(z), z p^{\prime}(z), z^{2} p^{\prime \prime}(z) ; z\right)=\phi\left(\mathcal{J}_{s+1, b}^{t} f(z), \mathcal{J}_{s, b}^{t}(z), \mathcal{J}_{s-1, b}^{t}(z) ; z\right)
$$

Therefore, we can rewrite (2.18) as

$$
\psi\left(p(z), z p^{\prime}(z), z^{2} p^{\prime \prime}(z) ; z\right) \in \Omega
$$

Then the proof is completed by showing that the admissibility condition for $\phi \in \Phi_{1}[\Omega, q]$ is equivalent to the admissibility condition for $\Psi$ as given in Definition 1.3.

Since

$$
\frac{\tau}{s}+1=\frac{(t+b)^{2} w-(t+b-1)^{2} u}{(t+b) v-(t+b-1) u}-2(t+b-1)
$$

Therefore, $\psi \in \Psi[\Omega, q]$. Also, by Theorem 1.1, $p(z) \prec q(z)$.

If $\Omega \neq \mathbb{C}$ is a simply connected domain, then $\Omega=h(\mathbb{U})$ for some conformal mapping $h(z)$ of $\mathbb{U}$ onto $\Omega$. In this case, the class $\Phi_{1}[h(\mathbb{U}), q]$ is written as $\Phi_{1}[h, q]$.

In the particular case $q(z)=M z, M>0$, the class of admissible functions $\Phi_{1}[\Omega, q]$ is denoted by $\Phi_{1}[\Omega, M]$.

The following theorem is a direct consequence of Theorem 2.5.

Theorem 2.6 Let $\phi \in \Phi_{1}[h, q]$. If $f(z) \in A(p)$ satisfies the subordination relation

$$
\phi\left(\frac{\mathcal{J}_{s+1, b}^{t}(z)}{z^{p-1}}, \frac{\mathcal{J}_{s, b}^{t}(z)}{z^{p-1}}, \frac{\mathcal{J}_{s-1, b}^{t}(z)}{z^{p-1}} ; z\right) \prec h(z) \quad(z \in \mathbb{U})
$$

then

$$
\frac{\mathcal{J}_{s+1, b}^{t}}{z^{p-1}} \prec q(z)
$$

Definition 2.4 Let $\Omega$ be a set in $\mathbb{C}$ and $M>0$. The class of admissible functions $\Phi_{1}[\Omega, M]$ consists of those functions $\phi: \mathbb{C}^{3} \times \mathbb{U} \rightarrow \mathbb{C}$ that satisfy the admissibility condition

$$
\phi\left(M e^{i \theta}, \frac{k+t+b-1}{t+b} M e^{i \theta}, \frac{L+\left((2 t+2 b-1) k+(t+b-1)^{2}\right) M e^{i \theta}}{(t+b)^{2}} ; z\right) \notin \Omega,
$$

where $z \in \mathbb{U}$ and $\operatorname{Re}\left(L e^{-i \theta}\right) \geq(k-1) k M$ for all real $\theta$ and $k \geq 1$. 
Corollary 2.5 Let $\phi \in \Phi_{1}[\Omega, M]$. If $f(z) \in A(p)$ satisfies

$$
\phi\left(\frac{\mathcal{J}_{s+1, b}^{t}(z)}{z^{p-1}}, \frac{\mathcal{J}_{s, b}^{t}(z)}{z^{p-1}}, \frac{\mathcal{J}_{s-1, b}^{t}(z)}{z^{p-1}} ; z\right) \in \Omega \quad(z \in \mathbb{U})
$$

then

$$
\left|\frac{\mathcal{J}_{s+1, b}^{t}(z)}{z^{p-1}}\right|<M
$$

In the case $\Omega=q(\mathbb{U})=\{\omega:|w|<M\}$, for simplification we denote by $\Phi_{1}[M]$ to the class $\Phi_{1}[\Omega, M]$.

Corollary 2.6 Let $\phi \in \Phi_{1}[M]$. If $f(z) \in A(p)$ satisfies

$$
\left|\phi\left(\frac{\mathcal{J}_{s+1, b}^{t}(z)}{z^{p-1}}, \frac{\mathcal{J}_{s, b}^{t}(z)}{z^{p-1}}, \frac{\mathcal{J}_{s-1, b}^{t}(z)}{z^{p-1}} ; z\right)\right|<M \quad(z \in \mathbb{U}),
$$

then

$$
\left|\frac{\mathcal{J}_{s+1, b}^{t}(z)}{z^{p-1}}\right|<M
$$

Corollary 2.7 If $f(z) \in A(p)$ and $\left|\frac{\mathcal{J}_{s, b}^{t}(z)}{z^{p-1}}\right|<M$. Then

$$
\left|\frac{\mathcal{J}_{s+n, b}^{t}(z)}{z^{p-1}}\right|<M \quad(n \in \mathbb{Z}, z \in \mathbb{U})
$$

Proof Putting $\phi(u, v, w ; z)=v$, in Corollary 2.6, we have

$$
\left|\frac{\mathcal{J}_{s, b}^{t}(z)}{z^{p-1}}\right|<M \quad \Rightarrow \quad\left|\frac{\mathcal{J}_{s+1, b}^{t}(z)}{z^{p-1}}\right|<M
$$

Therefore, the result is obtained by induction.

Corollary 2.8 Let $M>0$ and $\operatorname{Re}(b)>1-t$. If $f(z) \in A(p)$ satisfies

$$
\begin{aligned}
& \left|(t+b-1)^{2} \frac{\mathcal{J}_{s+1, b}^{t}(z)}{z^{p-1}}+(t+b) \frac{\mathcal{J}_{s, b}^{t}(z)}{z^{p-1}}-(t+b)^{2} \frac{\mathcal{J}_{s-1, b}^{t}(z)}{z^{p-1}}\right| \\
& \quad<M(t-1+\operatorname{Re}(b)),
\end{aligned}
$$

then

$$
\left|\frac{\mathcal{J}_{s+1, b}^{t}(z)}{z^{p-1}}\right|<M
$$

Proof In Corollary 2.5, taking $\phi(u, v, w ; z)=(t+b-1)^{2} u-(t+b) v-(t+b)^{2} w$ and $\Omega=h(\mathbb{U})$ where $h(z)=[(t-1+\operatorname{Re}(b))] M z$. 
Since

$$
\begin{aligned}
& \left|\phi\left(M e^{i \theta}, \frac{k+t+b-1}{t+b} M e^{i \theta}, \frac{L+\left((2 t+2 b-1) k+(t+b-1)^{2}\right) M e^{i \theta}}{(t+b)^{2}} ; z\right)\right| \\
& \quad=\left|(t+b-1)^{2} M e^{i \theta}-(k+t+b-1) M e^{i \theta}-\left[L+\left((2 t+2 b-1) k+(t+b-1)^{2}\right) M e^{i \theta}\right]\right| \\
& \quad=\left|L+(2 k-1)(t+b-1) M e^{i \theta}\right| \\
& \quad \geq \operatorname{Re}\left(L e^{-i \theta}\right)+(2 k-1) M \operatorname{Re}(t+b-1) \\
& \quad \geq k(k-1) M+(2 k-1) M(t-1+\operatorname{Re}(b)) \\
& \quad \geq M(t-1+\operatorname{Re}(b)) .
\end{aligned}
$$

Therefore, $\phi \in \Phi[\Omega, M]$ satisfies the admissible condition (2.14). Then we have the theorem by Corollary 2.5 .

Definition 2.5 Let $\Omega$ be a set in $\mathbb{C}$ and $q(z) \in \mathbb{D} \cap A_{p}$. The class of admissible functions $\Phi_{2}[\Omega, q]$ consists of those functions $\phi: \mathbb{C}^{3} \times \mathbb{U} \rightarrow \mathbb{C}$ that satisfy the admissibility condition

$$
\phi(u, v, w ; z) \notin \Omega
$$

whenever

$$
\begin{aligned}
& u=q(\varsigma), \quad v=q(\varsigma)+\frac{k \varsigma q^{\prime}(\varsigma)}{(t+b) q(\varsigma)} \quad(q(\varsigma) \neq 0) \\
& \operatorname{Re}\left(\frac{(t+b) v(w-v)-(t+b)(v-u)(2 u-v)}{(v-u)}\right) \geq k \operatorname{Re}\left(\frac{\varsigma q^{\prime \prime}(\varsigma)}{q^{\prime}(\varsigma)}+1\right)
\end{aligned}
$$

where $z \in \mathbb{U}, \zeta \in \partial \mathbb{U} \backslash E(q)$ and $k \geq 1$.

$$
(z \in \mathbb{U} ; \zeta \in \partial \mathbb{U} \backslash E(q) ; k \geq 1)
$$

Theorem 2.7 Let $\phi \in \Phi_{2}[\Omega, q]$ and $\mathcal{J}_{s+1, b}^{t}(z) \neq 0$. If $f(z) \in A_{p}$ satisfies

$$
\left\{\phi\left(\frac{\mathcal{J}_{s, b}^{t}(z)}{\mathcal{J}_{s+1, b}^{t}(z)}, \frac{\mathcal{J}_{s-1, b}^{t}(z)}{\mathcal{J}_{s, b}^{t}(z)}, \frac{\mathcal{J}_{s-2, b}^{t}(z)}{\mathcal{J}_{s-1, b}^{t}(z)} ; z\right)\right\} \subset \Omega \quad(z \in \mathbb{U})
$$

then

$$
\frac{\mathcal{J}_{s, b}^{t}(z)}{\mathcal{J}_{s+1, b}^{t}(z)} \prec q(z)
$$

Proof Let us define the analytic function $p(z)$ as

$$
p(z)=\frac{\mathcal{J}_{s, b}^{t}(z)}{\mathcal{J}_{s+1, b}^{t}(z)} \quad(z \in \mathbb{U})
$$

Using (2.4) and (2.34), we get

$$
\frac{\mathcal{J}_{s-1, b}^{t}(z)}{\mathcal{J}_{s, b}^{t}(z)}=p(z)+\frac{1}{(t+b)} \frac{z p^{\prime}(z)}{p(z)}
$$


which implies

$$
\frac{\mathcal{J}_{s-2, b}^{t}(z)}{\mathcal{J}_{s-1, b}^{t}(z)}=p(z)+\frac{1}{(t+b)}\left\{\frac{z p^{\prime}(z)}{p(z)}+\frac{(t+b) z p^{\prime}(z)+\frac{z^{2} p^{\prime \prime}(z)}{p(z)}+\frac{z p^{\prime}(z)}{p(z)}-\left(\frac{z p^{\prime}(z)}{p(z)}\right)^{2}}{(t+b) p(z)+\frac{z p^{\prime}(z)}{p(z)}}\right\} .
$$

Let us define the parameters $u, v$ and $w$ as

$$
\begin{aligned}
& u=r, \quad v=r+\frac{1}{(t+b)} \frac{s}{r} \text { and } \\
& w=r+\frac{1}{(t+b)}\left\{\frac{s}{r}+\frac{(t+b) s+\frac{\tau}{r}+\frac{s}{r}-\left(\frac{s}{r}\right)^{2}}{(t+b) r+\frac{s}{r}}\right\} .
\end{aligned}
$$

Now, we define the transformation

$$
\begin{aligned}
& \psi: \mathbb{C}^{2} \times \mathbb{U} \rightarrow \mathbb{C} \\
& \psi(r, s, \tau ; z)=\phi(u, v, w ; z),
\end{aligned}
$$

by using the relations (2.34), (2.35), (2.36) and (2.38), we have

$$
\psi\left(p(z), z p^{\prime}(z), z^{2} p^{\prime \prime}(z) ; z\right)=\phi\left(\frac{\mathcal{J}_{s, b}^{t}(z)}{\mathcal{J}_{s+1, b}^{t}(z)}, \frac{\mathcal{J}_{s-1, b}^{t}(z)}{\mathcal{J}_{s, b}^{t}(z)}, \frac{\mathcal{J}_{s-2, b}^{t}(z)}{\mathcal{J}_{s-1, b}^{t}(z)} ; z\right) .
$$

Therefore, we can rewrite (2.32) as

$$
\psi\left(p(z), z p^{\prime}(z), z^{2} p^{\prime \prime}(z) ; z\right) \in \Omega
$$

Then the proof is completed by showing that the admissibility condition for $\phi \in \Phi_{2}[\Omega, q]$ is equivalent to the admissibility condition for $\Psi$ as given in Definition 1.3.

Since

$$
\frac{\tau}{s}+1=\frac{(t+b) v(w-v)-(t+b)(v-u)(2 u-v)}{(v-u)} .
$$

Therefore, $\psi \in \Psi[\Omega, q]$. Also, by Theorem 1.1, $p(z) \prec q(z)$.

If $\Omega \neq \mathbb{C}$ is a simply connected domain, then $\Omega=h(\mathbb{U})$ for some conformal mapping $h(z)$ of $\mathbb{U}$ onto $\Omega$. In this case the class $\Phi_{2}[h(\mathbb{U}), q]$ is written as $\Phi_{2}[h, q]$.

In the particular case $q(z)=1+M z, M>0$, the class of admissible functions $\Phi_{2}[\Omega, q]$ is denoted by $\Phi_{2}[\Omega, M]$.

The following theorem is a direct consequence of Theorem 2.7.

Theorem 2.8 Let $\phi \in \Phi_{2}[h, q]$.If $f(z) \in A(p)$ satisfies the subordination relation

$$
\phi\left(\frac{\mathcal{J}_{s, b}^{t}(z)}{\mathcal{J}_{s+1, b}^{t}(z)}, \frac{\mathcal{J}_{s-1, b}^{t}(z)}{\mathcal{J}_{s, b}^{t}(z)}, \frac{\mathcal{J}_{s-2, b}^{t}(z)}{\mathcal{J}_{s-1, b}^{t}(z)} ; z\right) \prec h(z) \quad(z \in \mathbb{U})
$$

then

$$
\frac{\mathcal{J}_{s, b}^{t}(z)}{\mathcal{J}_{s+1, b}^{t}(z)} \prec q(z)
$$


Definition 2.6 Let $\Omega$ be a set in $\mathbb{C}$ and $M>0$. The class of admissible functions $\Phi_{2}[\Omega, M]$ consists of those functions $\phi: \mathbb{C}^{3} \times \mathbb{U} \rightarrow \mathbb{C}$ that satisfy the admissibility condition

$$
\begin{aligned}
\phi\left(1+M e^{i \theta}, 1+\left(1+\frac{k}{(t+b)\left(1+M e^{i \theta}\right)}\right) M e^{i \theta}, 1+\left(1+\frac{k}{(t+b)\left(1+M e^{i \theta}\right)}\right) M e^{i \theta}\right. \\
\left.+\frac{\left(M+e^{-i \theta}\right)\left[L e^{-i \theta}+(t+b+1) k M+(t+b) k M^{2} e^{i \theta}\right]-k^{2} M}{(t+b)\left(M+e^{-i \theta}\right)\left[(t+b) e^{-i \theta}+(2(t+b)+k) M+(t+b) M^{2} e^{i \theta}\right]} ; z\right) \\
\in \Omega,
\end{aligned}
$$

where $z \in \mathbb{U}$ and $\operatorname{Re}\left(L e^{-i \theta}\right) \geq(k-1) k M$ for all real $\theta$ and $k \geq 1$.

Corollary 2.9 Let $\phi \in \Phi_{2}[\Omega, M]$. Iff $(z) \in A(p)$ satisfies

$$
\phi\left(\frac{\mathcal{J}_{s, b}^{t}(z)}{\mathcal{J}_{s+1, b}^{t}(z)}, \frac{\mathcal{J}_{s-1, b}^{t}(z)}{\mathcal{J}_{s, b}^{t}(z)}, \frac{\mathcal{J}_{s-2, b}^{t}(z)}{\mathcal{J}_{s-1, b}^{t}(z)} ; z\right) \in \Omega \quad(z \in \mathbb{U})
$$

then

$$
\left|\frac{\mathcal{J}_{s, b}^{t}(z)}{\mathcal{J}_{s+1, b}^{t}(z)}\right|<1+M
$$

In the case $\Omega=q(\mathbb{U})=\{\omega:|\omega-1|<M\}$, for simplification, we denote by $\Phi_{2}[M]$ to the class $\Phi_{2}[\Omega, M]$.

Corollary 2.10 Let $\phi \in \Phi_{2}[M]$. If $f(z) \in A(p)$ satisfies

$$
\left|\phi\left(\frac{\mathcal{J}_{s, b}^{t}(z)}{\mathcal{J}_{s+1, b}^{t}(z)}, \frac{\mathcal{J}_{s-1, b}^{t}(z)}{\mathcal{J}_{s, b}^{t}(z)}, \frac{\mathcal{J}_{s-2, b}^{t}(z)}{\mathcal{J}_{s-1, b}^{t}(z)} ; z\right)-1\right|<M \quad(z \in \mathbb{U}),
$$

then

$$
\left|\frac{\mathcal{J}_{s, b}^{t}(z)}{\mathcal{J}_{s+1, b}^{t}(z)}-1\right|<M
$$

Corollary 2.11 Let $M>0$. Iff $(z) \in A(p)$ satisfies

$$
\begin{array}{r}
\left|\frac{\mathcal{J}_{s-1, b}^{t}(z)}{\mathcal{J}_{s, b}^{t}(z)}-\frac{\mathcal{J}_{s, b}^{t}(z)}{\mathcal{J}_{s+1, b}^{t}(z)}\right| \\
\quad<\frac{M}{(1+M)(1+|b|)},
\end{array}
$$

then

$$
\left|\frac{\mathcal{J}_{s, b}^{t}(z)}{\mathcal{J}_{s+1, b}^{t}(z)}-1\right|<M
$$

Proof In Corollary 2.9, taking $\phi(u, v, w ; z)=u-v$ and $\Omega=h(\mathbb{U})$ where $h(z)=\frac{M z}{(1+M)(1+|b|)}$. 
Since

$$
\begin{aligned}
|\phi(u, v, w ; z)| & =\left|\left(\frac{k}{(t+b)\left(1+M e^{i \theta}\right)}\right) M e^{i \theta}\right| \\
& =\left|\left(\frac{k}{(t+b)\left(1+M e^{i \theta}\right)}\right)\right| M \\
& >\frac{M}{(1+M)(1+|b|)} .
\end{aligned}
$$

Therefore, $\phi \in \Phi[\Omega, M]$ satisfies the admissible condition (2.41). Then we have the theorem by Corollary 2.11 .

\section{Competing interests}

The authors declare that they have no competing interests.

\section{Authors' contributions}

All authors contributed equally to the manuscript. Also, all authors have read and approved the final version of the manuscript.

\section{Author details}

${ }^{1}$ Department of Mathematics, Faculty of Science, University of Mansoura, Mansoura, 35516, Egypt. ${ }^{2}$ Current address: Department of Mathematics, Faculty of Science, University of Hail, Hail, Saudi Arabia. ${ }^{3}$ Department of Mathematics, College of Science, King Khaled University, P.O. Box 9004, Abha, 61431, Saudi Arabia. ${ }^{4}$ Current address: Department of Mathematics, Faculty of Science, Jazan University, P.O. Box 277, Jazan, 45142, Saudi Arabia.

\section{Received: 8 February 2013 Accepted: 4 April 2013 Published: 17 April 2013}

\section{References}

1. Srivastava, HM, Choi, J: Series Associated with the Zeta and Related Functions. Kluwer Academic, Dordrecht (2001)

2. Choi, J, Jang, DS, Srivastava, HM: A generalization of the Hurwitz-Lerch Zeta function. Integral Transforms Spec. Funct. 19(1-2), 65-79 (2008)

3. Ferreira, C, López, JL: Asymptotic expansions of the Hurwitz-Lerch zeta function. J. Math. Anal. Appl. 298, 210-224 (2004)

4. Gupta, PL, Gupta, RC, Ong, S, Srivastava, HM: A class of Hurwitz-Lerch zeta distributions and their applications in reliability. Appl. Math. Comput. 196(2), 521-531 (2008)

5. Luo, QM, Srivastava, HM: Some generalizations of the Apostol-Bernoulli and Apostol-Euler polynomials. J. Math. Anal. Appl. 308, 290-302 (2005)

6. Kutbi, MA, Attiya, AA: Differential subordination result with the Srivastava-Attiya integral operator. J. Inequal. Appl. 2010, 1-10 (2010)

7. Kutbi, MA, Attiya, AA: Differential subordination results for certain integrodifferential operator and it's applications. Abstr. Appl. Anal. 2012, 638234 (2012)

8. Srivastava, HM, Attiya, AA: An integral operator associated with the Hurwitz-Lerch zeta function and differential subordination. Integral Transforms Spec. Funct. 18(3-4), 207-216 (2007)

9. Owa, S, Attiya, AA: An application of differential subordinations to the class of certain analytic functions. Taiwan. J. Math. 13(2A), 369-375 (2009)

10. Alexander, JW: Functions which map the interior of the unit circle upon simple region. Ann. Math. 17, 12-22 (1915)

11. Libera, RJ: Some classes of regular univalent functions. Proc. Am. Math. Soc. 16, 755-758 (1965)

12. Bernardi, SD: Convex and starlike univalent functions. Transl. Am. Math. Soc. 135, 429-449 (1969)

13. Jung, JB, Kim, YC, Srivastava, HM: The Hardy space of analytic functions associated with certain one-parameter families of integral operator. J. Math. Anal. Appl. 176, 138-147 (1993)

14. Cho, NE, Kim, IH, Srivastava, HM: Sandwich-type theorems for multivalent functions associated with the Srivastava-Attiya operator. Appl. Math. Comput. 217(2), 918-928 (2010)

15. Liu, J-L: Subordinations for certain multivalent analytic functions associated with the generalized Srivastava-Attiya operator. Integral Transforms Spec. Funct. 19(11-12), 893-901 (2008)

16. Noor, Kl, Bukhari, SZ: Some subclasses of analytic and spiral-like functions of complex order involving the Srivastava-Attiya integral operator. Integral Transforms Spec. Funct. 21(12), 907-916 (2010)

17. Elrifai, EA, Darwish, HE, Ahmed, AR: Some applications of Srivastava-Attiya operator to $p$-valent starlike functions. J. Inequal. Appl. 2010, 1-11 (2010)

18. Miller, SS, Mocanu, PT: Differential Subordinations: Theory and Applications. Series in Pure and Applied Mathematics, vol. 225. Dekker, New York (2000) 